\title{
„Gemeinsames Erzählen“ und Prozesse der Wirklichkeitskonstruktion in familiengeschichtlichen Gesprächen
}

\section{Bruno Hildenbrand, Walther Jahn}

Fachbereich Gesellschaftswissenschaften, Johann Wolfgang Goethe-Universität, Senckenberganlage 15, D-6000 Frankfurt/Main

Zu s a m m e nf a ssu ng: In diesem Aufsatz wird das, gemeinsame familiengeschichtliche Gespräch" vorgeschlagen als eine Methode, Familienbiographien und aktuelle Sinnbildungsprozesse in Familien zugleich zu erheben. Anhand eines Fallbeispiels wird ein Analyseverfahren demonstriert, in welchem sequenzanalytisch die Vorgehensweise der "Strukturalen Hermeneutik“ (Oevermann) übernommen und eine Brücke zur „Grounded Theory” (Glaser/Strauss) geschlagen wird. Die bei diesen Verfahren vorliegende enge Verzahnung von Methode und materialer Analyse wird erkenntlich daran, daß das Thema des gemeinsamen familiengeschichtlichen Erzählens im Kontext der Analyse von Modernisierungsprozessen in bäuerlichen Familienbetrieben entfaltet wird.

\section{1. „Gemeinsames Erzählen" und Familiengeschichte}

In einem Forschungsprojekt über Familien Schizophrener (Blankenburg, Hildenbrand 1983) arbeiteten wir mit Hilfe ethnographischer Verfahren fallrekonstruktiv Typen alltagsweltlicher Orientierung heraus. Einer der von uns benutzten methodischen Zugänge war das familiengeschichtliche Gespräch. Im Verlauf von Datenerhebung und -analyse entdeckten wir in diesen Familiengesprächen Interaktions- und Sinnbildungsprozesse, deren Erkenntniswert über das hinausging, was wir von diesen Gesprächen erwartet hatten. Wir beobachteten, daß die Beteiligten nicht nur über ihre Wirklichkeit berichteten, sondern diese situativ handelnd konstruierten und modifizierten und daß dabei Aspekte familienspezifischer Weltsichten zum Ausdruck kamen, die den Alltag dieser Familien durchgängig strukturieren. In dem Projekt war es allerdings nicht möglich, die vorhandenen familiengeschichtlichen Gespräche auf die diesem Phänomen zugrundeliegenden Prozesse, insbesondere auf die interaktiven Muster in diesen Familiengesprächen hin genauer zu untersuchen. Dieses Thema haben wir zum Gegenstand eines eigenen Forschungsprojekts gemacht. ${ }^{1}$ Wir werden im folgenden theoretische und methodische Fragen des gemeinsamen Erzählens an dem für dieses Thema besonders geeigneten Fall familiengeschichtlicher

\footnotetext{
' DFG-Projekt „Prozesse der Wirklichkeitskonstruktion beim familiengeschichtlichen Erzählen“, Projektleiter B. Hildenbrand und U. Oevermann, unter Mitarbeit von W. Jahn, H. Müller, R. Schmitt sowie H. HennesDoh, S. Hilbert, B. Kayser, M. Lang, D. ThomaRadler.
}

Gespräche erörtern und eine exemplarische Textanalyse vorstellen.

Zunächst ist die Frage zu klären, in welcher Weise man von gemeinsamem familiengeschichtlichen Erzählen sprechen kann. ${ }^{2}$ In seinem Buch über einfache Formen des Erzählens bespricht Jolles (1982, ursprünglich 1930) die Sage.

Jolles rechnet diese Erzählform einer Gesellschaftsformation zu, in welcher sich Wirklichkeit ,als Familie aufbaut, in der sie in ihrer Ganzheit nach dem Begriff des Stammes, des Stammbaums, der Blutsverwandtschaft gedeutet wird." (1982: 74) Geschichte kommt in der Sage als "festgefügte, in sich geschlossene mündliche Erzählung" (1982: 66) eines Familiengeschehens vor. Sagen sind typisch für Sozialzusammenhänge, welche von einer „urtümlichen Zweiteilung der Welt, die der angestammten und vertrauten Heimat alles Übrige als zweitrangiges Draußen gegenüberstellt" ausgehen. Es handelt sich dabei um eine Welt, ,die geprägt ist durch ihre Überschaubarkeit, ihre Enge und Tiefe“. (Bausinger 1958, S. 247)

Obwohl Bausinger (mit Jolles) der Ansicht ist, daß Familiensagen im Erörtern von Verwandtschaftsbeziehungen auch heute noch lebendig sind, stellt er jedoch fest, daß die Sage im alltäglichen Erzählen keine große Bedeutung mehr hat, weil aufgrund der historischen Entwicklung, insbesondere von „Technik und Verkehr“ (1958: 257) die ursprüngliche Zweiteilung von Heimat und Fremde aufgehoben werde.

Bausinger spricht von einem Proze $B$, der mit dem Hinweis auf Technik und Verkehr nicht zurei-

${ }^{2}$ Für die Überlegungen in diesem Abschnitt waren uns Hinweise von J. R. Bergmann, Konstanz, sehr hilfreich, insbesondere sein Verweis auf Jolles und dessen Bedeutung für das vorliegende Thema. 
chend erhellt ist, in welchem vielmehr diese Phänomene Aspekte umfassender Veränderungen innerhalb der Entwicklung zur Moderne sind. Mit der im Verlauf der Industrialisierung sich beschleunigenden institutionellen Differenzierung sind die einzelnen Lebensbereiche der Individuen Veränderungen unterworfen, die nicht nur in der sukzessiven Trennung von öffentlicher und privater Sphäre zu beobachten sind, sondern auch innerhalb dieser Sphären zu sehen sind an dem zunehmenden Zwang zur Reflexion alltäglicher Orientierung angesichts der grundsätzlichen Fremdheit vieler institutioneller Bereiche. Für das Individuum erhöht sich damit die Notwendigkeit eigener Orientierungsleistungen zur Selbstvergewisserung seines sozialen Orts. Der privaten Sphäre und hier hauptsächlich Ehe und Familie kommt die Aufgabe zu, Orientierungsmodelle zu entwikkeln und dies ist auch der Bereich, in welchem Familiengeschichten für die soziale Konstruktion von Wirklichkeit von Bedeutung sind. (Berger, Kellner 1965)

In der volkskundlichen Literatur wird auch heute noch verschiedentlich der Begriff der Sage benutzt, wenn es um gemeinsames familiengeschichtliches Erzählen geht. ${ }^{3}$ In den dort analysierten Familiengeschichten ist allerdings eine andere Form von Familiensage enthalten als jene, die mit diesem Begriff bezeichnet wird. Es geht vielmehr um das, was Jolles als Memorabile bezeichnet hat. Diese einzelnen Geschichten haben die traditionalen Formen alltäglichen Erzählens (Märchen, Sage, Schwank) abgelöst.

Jolles (1982) betrachtet die Moderne als die „Welt des Tatsächlichen", als eine Welt von ununterscheidbaren Tatbeständen, in welchem dem Memorabile die Aufgabe zukomme, „diese ununterschiedliche Welt zu scheiden, zu unterscheiden und konkret werden zu lassen" (1982: 12) und ihr dadurch (und nur dadurch) „Glaubwürdigkeit" zu verleihen. Das Memorabile selegiert aus der ununterscheidbaren Welt: „Der Ausschnitt ist bestrebt, aus dem allgemeinen Geschehen etwas einmalig herauszuheben, das als Ganzes den Sinn dieses Geschehens bedeutet; in diesem Ganzen sind die Einzelheiten in einer Weise angeordnet, daß sie einzeln, in ihren Beziehungen, in ihrer Gesamtheit erklärend, erörternd, ver-

${ }^{3}$ Boatright (1958) 2. B. schreibt: „Ich benutze diesen Begriff hauptsächlich, um überliefertes Wissen $\mathrm{zu}$ kennzeichnen, das sich um Familien herum ansammelt, welches durch mündliche Wiedergabe bewahrt und verändert wird und welches für wahr gehalten wird. Diese cluster von Geschichten bilden niemals eine verbundene Geschichte."(1958: 1) gleichend und gegenüberstellend den Sinn des Geschehens hervorheben." (1982: 203)

Familiengeschichte wäre demnach in der Moderne nicht mehr tradiert in der Form von Familiensagas, sondern sie wäre aufgelöst in einzelne unverbundene Geschichten, in denen die Gesamtheit, oder: die Struktur einer Familie verdichtet zum Ausdruck kommt. ${ }^{4}$

Wir können daher annehmen, daß die aktuelle Form familiengeschichtlichen Erzählens die des Memorabiles ist. Das heißt, daß in Familien einzelne, nicht in einem systematischen Zusammenhang stehende Familiengeschichten erzählt werden, die die Struktur der erzählenden Familie zum Ausdruck bringen, wobei umgekehrt die Familienstruktur die Grundlage dafür legt, welche Geschichten erzählt werden und wie das geschieht.

Erste Analysen (Hildenbrand und Oevermann 1987) von Familiengeschichten, welche wir in bäuerlichen Familien durchgeführt haben, weisen jedoch darauf hin, daß tatsächlich übergreifende Erzähllinien feststellbar sind. Diese sind das Ergebnis individueller Leistungen einzelner Erzähler, welche als ,Experten der Familiengeschichte ' gelten können. Damit wäre die Familiengeschichte nicht mehr unbefragtes, allen zuhandenes Wissen als Rahmen für Sinngebung, sondern mit einer paradoxen Formulierung als, individuell hergestellte Familiensaga' zu bezeichnen.

Lehmann (1978), der sich mit der Bedeutung von Geschichten aus dem eigenen Leben, die im Alltag erzählt werden, befaßt hat, führt zwei Gesichtspunkte aus, die für solche Geschichten von Bedeutung sind: Sie dienen dazu, ihrem Erzähler in

\footnotetext{
${ }^{4}$ Vgl. dazu Koselleck (1979). Für ihn leitet die Geschichte „,ihre Lehren nicht nur aus Geschichten ab, sondern ebenso aus den ,Bewegungsstrukturen' unserer Geschichte“ (1979: 157). Ereignisse, welche in Geschichten erzählbar sind, werden in ihrem Verlauf durch strukturelle Bedingungen ermöglicht, umgekehrt sind „Strukturen nur greifbar im Medium von Ereignissen, in derien sich Strukturen artikulieren" (1979: 149). Allerdings bestehe zwischen Ereignis und Struktur ein Hiatus, da beide sich in unterschiedlichen Zeithorizonten bewegen. Anders als Koselleck betont Jolles nur die eine Seite des Wechselverhältnisses von Struktur und Ereignis: die Erzählung eines Ereignisses in der Form des Memorabiles macht den Sinn des Geschehensganzen (der Struktur) sichtbar. Koselleck (1979) fügt dem hinzu, daß der Ausschnitt aus dem Geschehensganzen in Form einer Erzählung eines Ereignisses nicht von ungefähr kommt, sondern daß er durch eine Struktur motiviert ist.
} 
settings, die über eine gemeinsame - lebenspraktisch hergestellte - Geschichte erstens den egalisierenden Tendenzen der Gruppe entgegenzuwirken und erzählend die eigene Individualität zu sichern, und zweitens sich gleichzeitig der Solidarität der Gruppe zu vergewissern. Oft bestehe eine „fein abgestimmte Gleichgewichtsbeziehung von solidarisierenden und individualisierenden Geschichten“ (Lehmann 1978: 212) innerhalb von Erzählungen im Alltag.

Neben solidarisierenden und individualisierenden Geschichten sind die von Lehmann untersuchten Rechtfertigungsgeschichten, die im Familienverband erzählt werden, für unsere Zwecke besonders interessant. Diese dienen dem Erzähler dazu, „seiner Umwelt akzeptable Versionen für bestimmte lebensgeschichtliche Perioden und Vorkommnisse anzubieten“ (1980: 57). Lehmann zeigt hier, daß Rechtfertigungsgeschichten in Familien eine solidarisierende Funktion haben können. Insbesondere würden sie Familienthemen - im Sinne von Hess und Handel (1975) - in deren Bearbeitung die Familie ihre Wirklichkeit konstruiert, konsistent machen. ${ }^{5}$

\section{Die Familie als Erfahrungs-, Erinnerungs- und Erzählgemeinschaft und als Ort von Selbstthematisierung}

Natalia Ginzburg schreibt in ihrem Roman ,Mein Familienlexikon“:

„Wir sind fünf Geschwister. Wir wohnen in verschiedenen Städten, einige sogar im Ausland, und wir schreiben uns nicht häufig. Wenn wir uns treffen, sind wir den anderen gegenüber manchmal vielleicht zerstreut oder gleichgültig. Doch ein Wort genügt zwischen uns. Ein Wort oder ein Satz genügt: Einer jener Sätze, die uns, als wir Kinder waren, unendliche Male wiederholt wurden. Es genügt, uns zu sagen: Wir sind nicht nach Bergamo gekommen, um einen Ausflug zu machen oder: wonach stinkt der Schwefelwasserstoff? um mit einem Schlag

\footnotetext{
${ }^{5}$ Auch Quasthoff hat gemeinsames Erzählen bei Ehepaaren untersucht. Kurz gesagt, stehen Lehmanns und Quasthoffs Arbeiten in einem gegensätzlichen Verhältnis zueinander dergestalt, daß Lehmann eigenerlebte, individuell erzählte Geschichten erhebt und analysiert und dabei zur sozialen Fundiertheit dieses Vorgangs gelangt, während Quasthoff explizit beim gemeinsamen Geschichtenerzählen zwar ihren Ausgang nimmt, dies dann aber auflöst in eine Addition individuell erzählter Geschichten. Letztlich ist Quasthoffs Erzählbegriff kognitivistisch und individualistisch und daher für unsere Zwecke wenig hilfreich.
}

unsere alten Beziehungen, unsere Kindheit und Jugend wiederzufinden, die untrennbar mit diesen Sätzen, mit diesen Worten verbunden ist. An einem dieser Worte würden wir uns im Dunkeln einer Grotte unter Millionen von Menschen als Geschwister wiedererkennen. Diese Sätze sind unser Latein, das Vokabular unserer vergangenen Tage, sie sind wie die Hieroglyphen der Ägypter oder Assyrer oder Babylonier; Zeugen einer Lebensgemeinschaft, die aufgehört hat zu sein, aber in Texten weiterlebt, die vor der Wut des Wassers und der Zerstörung der Zeit gerettet wurden. " (1983: 29f.)

Ginzburg spricht hier einen Aspekt von Familienleben an, welcher dessen unbefragte Sinnzusammenhänge umfaßt: die Milieuhaftigkeit von Familie (Gurwitsch 1977, Hildenbrand 1983). Für Gurwitsch bildet das Milieu einen Gesamtzusammenhang von habituell handelnden Individuen und von Gegenständen, wobei dieser Gesamtzusammenhang den Milieubeständen gegenüber Vorrang hat. Das Milieu setzt somit den Rahmen für Aufmerksamkeit und Interesse - einen Rahmen, den Halbwachs als kollektives bzw., soweit es um Familien geht, als Familiengedächtnis bezeichnet hat:

„Auf jeden Fall stellt das Familiengedächtnis aus verschiedenen aus der Vergangenheit behaltenen Elementen solcher Art (gemeint sind, Gewohnheiten und Denkweisen', ,die allgemeine Haltung der Gruppe', ,ihre Wesensart, Eigenheiten und Schwächen ${ }^{6}$ - die Verf.) einen Rahmen her, den es intakt zu halten versucht und der gewissermaßen zur traditionellen Ausrüstung der Familie gehört. Obwohl er aus datierbaren Fakten und Bildern besteht, die nur eine bestimmte Zeit gedauert haben, findet man doch Urteile der Familie selber über sie darin verwoben, und so teilt er auch die Wesensart dieser kollektiven Vorstellung, die sich nicht einem bestimmten Ort oder einem bestimmten Zeitmoment zuschreiben lassen, und die über dem Laufe der Zeit zu stehen scheinen." (1966: 210)

Daß Familie jedoch nicht nur als Ort unbefragter milieuhafter Sinnzusammenhänge begriffen werden kann, sondern auch als Ort von Selbstthematisierung, hat mit den eingangs erwähnten Prozessen des zunehmenden Auseinandertretens von Individuum und Gesellschaft zu tun. Für das Individuum bildet die Familie den Ort, an welchem sich Individuen als Individuen thematisieren, da identitätssichernde Milieus und Kontinuität gewährleistende, sozial verfügbare Identitätskonzepte zunehmend von Identitätsformationen abgelöst werden, die auf Selbstbeschreibungen und Selbstdarstellungen basieren und immer häufiger zur Vergewisserung lebensgeschichtlicher Entscheidungen herangezogen werden. (Brose und Hildenbrand 1988). Familie wäre dann als Bühne für solche individuellen Selbstdarstellungen zu betrachten. Die Familie 
kann weiterhin als Ort der Selbstthematisierung begriffen werden, an welchem sich die Familie als Familie thematisiert. Dies bedeutet, daß familiales Leben dauerhaft thematisiert und reflektiviert wird, während seine Dauer selbst grundsätzlich fragwürdig wird. ${ }^{6}$

Bei der Analyse gemeinsamen familiengeschichtlichen Erzählens ist daher Familie sowohl unter dem Aspekt von Milieuhaftigkeit als auch unter dem Aspekt reflexiver Selbstthematisierung in der genannten doppelten Weise zu betrachten. - Wie dies geschehen kann, möchten wir hier anhand der materialen Analyse eines Gesprächsausschnitts ansatzweise zeigen. Zuvor soll jedoch die methodische Vorgehensweise dargestellt werden.

\section{Die Erhebung und Analyse familiengeschichtlicher Gespräche}

\subsection{Eine Heuristik zur Untersuchung interaktiv realisierter Erzählungen}

Ein soziologisches Konzept gemeinsam realisierter Erzählungen befaßt sich in erster Linie mit den Interaktions- und Wissensstrukturen, in denen eine Aktualisierung von Erlebnissen und Erfahrungen stattfindet. In diesen Zusammenhang gehört auch die Frage nach dem Wozu der Erfahrungsaktualisierung. Die interaktive Einbettung bzw. die Pragmatik der Textproduktionssituation eröffnet Interaktionsspielräume, die das Verhältnis von $\mathrm{Er}$ fahrung und Darstellung strukturieren. Erst familiengeschichtliche Gespräche bieten im Gegensatz zu biographischen Erzählungen in Einzelinterviews die Möglichkeit, Interaktionsanalysen mit der Analyse von biographischen Prozessen zu verknüpfen. Dabei wird zunächst kein Unterschied zwischen ereignis-, erlebnis- oder erfahrungsbezogen realisierten Erzählungen gemacht, um einer immanenten Kategorienbildung am Datenmaterial nicht vorzugreifen und damit eine - in sprachwissenschaftlichen Untersuchungen weit verbreitete extern-klassifikatorische Vorgehensweise $\mathrm{zu}$ vermeiden.

Soll weder ein Begriff von ,Gemeinsamkeit' als formale Kategorie wie Lackmuspapier zur Bestimmung von Interaktionen im Erzählen verwendet werden noch ein bestimmter Begriff von ,Erzäh-

${ }^{6}$ Beck (1986: 118) zieht beide Aspekte, den von Familie als Diskursergebnis und den grundsätzlich fragwürdiger Dauer, zur Formel der „Verhandlungsfamilie auf Zeit" zusammen. lung", wie auch immer theoretisch angeleitet, einem Interaktionstext aufgepfropft werden, so kann nur eine genaue Analyse der sich in der Interaktion realisierenden Sinnstruktur zu einer soziologischen Typisierung von Erzählungen führen. Diese freilich wird nicht als Selbstzweck betrieben, sondern steht im Kontext materialer soziologischer Analysen. In unserem Fall handelt es sich um Untersuchungen zu Modernisierungsprozessen in der Landwirtschaft und ihrer Bedeutung für bäuerliche Familienwelten (siehe auch Hildenbrand 1988).

Die Konstitution einer Erzählgemeinschaft wird nicht als physisch oder formal definierte Beteiligung begriffen, sondern als interaktive Realisierung gemeinsamer Sinnhorizonte, kollektiver Erfahrungsmodi und gemeinsam gemachter Erfahrungen. Die Familie bildet dafür einen sinnvollen Bezugspunkt: Anhand dieser sozialen Einheit, welche Sozialität jeweils sowohl neu konstituiert als auch historisch tradiert, können synchrone und diachrone Perspektiven der Erfahrungskonstitution in ihrem Zusammenhang analysiert werden.

\subsection{Das empirische Material und die Erhebungssituation}

Volkskundler würden familiengeschichtliches Erzählen bei Geburtstagen, Verwandtenbesuchen, Familienfeiern und vergleichbaren Anlässen, in denen Erzählen in dieser Weise alltäglich ist, erheben. Dies bedingt jedoch lange Vorschaltphasen, in welchen sich die Forscher in und mit einem Untersuchungsfeld vertraut machen und über längere Zeiträume darin arbeiten. Wie kann nun der Sozialwissenschaftler solche Erzählungen erheben? In dem anfangs erwähnten Forschungsprojekt haben wir die Erfahrung gemacht, daß auch in Familiengesprächen, die zu sozialwissenschaftlichen Untersuchungszwecken geführt werden, solche alltäglich zuhandenen Familiengeschichten wie selbstverständlich einfließen. Voraussetzung dafür ist, da $\mathrm{B}$ die Sozialwissenschaftler als interessierte Fremde zuhören und sich nach Maßgabe des Gesprächs beteiligen. Das Verfahren des familiengeschichtlichen Gesprächs kann als forschungstechnische Abkürzung also durchaus auch hier eingesetzt werden.

Im Einzelnen gehen wir bei der Datenerhebung so vor:

(1) Das Familiengespräch findet bei der Familie zuhause statt. Es sollen möglichst alle zum Haushalt gehörenden Personen anwesend sein. Hierbei kommt es gelegentlich 
zu fallspezifisch motivierten Erweiterungen und Ausschlüssen von Gesprächsteilnehmern. Die in dem Familiengespräch nicht anwesenden Personen werden später zu Gesprächen direkt eingeladen, was, weil es den Ausschlußpraktiken der Familie zuwiderläuft, mit entsprechenden forschungspraktischen Problemen verbunden ist.

(2) Bei unseren bisherigen Familiengesprächen zeigte sich, daß die Teilnehmer ihre Beiträge oft geschlechtsspezifisch fokussieren. Wird nun das Gespräch von zwei Interviewern, einer Frau und einem Mann geführt, so wird eine größere Bandbreite von Äußerungen evoziert. Zudem können die Interviewer abwechselnd ihre Aufmerksamkeit auch auf gesprächsbegleitende Phänomene richten und diese dann im Anschluß an das Gespräch notieren.

(3) Die Gespräche werden auf Tonband aufgezeichnet. Die Geräte werden bei der telefonischen und/oder schriftlichen Vereinbarung des Gesprächs eingeführt, die Modi der Verwendung erläutert und die Techniken der Anonymisierung dargestellt.

(4) Zu Beginn des Gesprächs werden die Familienmitglieder aufgefordert, aus ihrem Familienleben von früher und heute zu erzählen. Von der Verwendung eines expliziten Erzählstimulus im Hinblick auf die Familiengeschichte haben wir Abstand genommen, da uns bei der Interpretation von zurückliegenden Familiengesprächen aufgefallen ist, daß dadurch unnötige Restriktionen der möglichen Themenvielfalt geschaffen werden. Die Durchführung des familiengeschichtlichen Gesprächs orientiert sich am Verlauf alltagsweltlicher Gespräche, wobei die Erfahrung mit den vorliegenden familiengeschichtlichen Gesprächen zeigt, daß methodisch angeleitete Interventionen überflüssig sind, weil das Gespräch von den Familienmitgliedern selbst gestaltet wird.

(5) Am Schluß des familiengeschichtlichen Gesprächs werden gemeinsam jene Sozialdaten der Familie anhand einer Kontrolliste ergänzt, welche während des $\mathrm{Ge}$ sprächs nicht genannt worden sind. Diese Ergänzung ermöglicht bei der Analyse auch einen Vergleich der im Erzählzusammenhang thematisierten Sozialdaten mit dem für eine zureichende Rekonstruktion der Familienstruktur und ihrer Entwicklung erforderlichen Grundbestand an Sozialdaten.

(6) Im Anschluß an das familiengeschichtliche Gespräch fertigen die Interviewer ein Beobachtungsprotokoll an. Dieses enthält als ergänzendes Datenmaterial die Entstehungsgeschichte des Gesprächs, eine Darstellung der Einbettung des Interviews in den räumlich-sozialen Lebenszusammenhang der Familie und eine Beschreibung des Hauses und seiner Einrichtung.

\subsection{Die Analyse des Datenmaterials}

Zunächst wird das Gespräch von den Interviewern selbst transkribiert. Die Transkription soll einerseits differenziert genug sein, um den Text nach formalen und textanalytischen Kriterien analysie- ren zu können, andererseits soll sie grob genug sein, um die anfallenden Datenmengen handhaben zu können und ein Mindestmaß an Übersichtlichkeit zu sichern.

Die Interpretation der Texte folgt dem Verfahren der sequenziellen Analyse. Die Sequenzanalyse kann als quasi-universelles Prinzip der interpretativen Sozialforschung angesehen werden. Allgemein gesprochen, ist die Sequenzanalyse die „Methodisierung der Idee einer sich im Interaktionsvollzug reproduzierenden sozialen Ordnung. " (Bergmann 1985: 313) Der in der Tradition des Symbolischen Interaktionismus stehende A. Strauss (1987) spricht von ,line by line-analysis". In der Objektiven Hermeneutik (vgl. U. Oevermann, T. Allert, E. Konau, J. Krambeck 1979 und U. Oevermann 1981, 1983) ist das Ziel der sequenzanalytischen Interpretation

„die schlüssige Rekonstruktion und Explikation des Zusammenhangs einer Strukturiertheit, der sich immer nur im sequentiellen Ablauf ihrer Reproduktion gültig erfassen läßt.“ (U. Oevermann 1983: 269)

Der für die Vorgehensweise zentrale Verfahrensschritt ist dabei:

„die Optionen oder Möglichkeiten des weiteren Verlaufs einer Interaktion an jeder Sequenzposition oder $\mathbf{Z u}$ standsstelle aufgrund einer extensiven Auslegung der objektiven Bedeutungsstrukturen zu bestimmen und dann die objektive Bedeutung der tatsächlich erfolgten nächsten Bewegung im Interaktionsablauf darauf abzubilden." (U. Oevermann 1981: 51)

Dieses Vorgehen zeigt so das Verhältnis von Allgemeinem und Besonderem in der sich individuierenden Fallstruktur auf.

„Die Fallspezifität bezeichnet ja zugleich die Reproduktionsgesetzlichkeit der Fallstruktur. In dem Maße, in dem in einer Sequenzanalyse mit fortschreitender Abarbeitung der Sequenzstellen der fallspezifische, d. h. die Reproduktionsgesetzlichkeit der Fallstruktur abbildende Ausschluß von Optionen systematisch freigelegt bzw. herausgeschnitten wird, liegt ein kumulatives Wissen über den inneren, interaktionsimmanenten Kontext der jeweils nächstfolgenden Äußerung vor und damit zugleich ein Wissen über jene fallspezifische innere Realität, die die Selektion unter den gleichermaßen der äußeren Realität gerecht werdenden Optionen systematisch motiviert." (Oevermann 1981: 54)

Es ist bereits angeklungen, daß sequenzanalytische Verfahren nicht beschränkt sind auf sprachliches (gesprochenes oder geschriebenes Material), wenn auch der Soziologe, da er mit Texten arbeitet, darauf angewiesen ist, außersprachliches Material zunächst zu versprachlichen, bevor er es analysieren kann: 
„Text ist, was der Fall ist, aber nicht alles, was der Fall ist, ist auch Text.“ (Soeffner 1980)

Handlungen sind als sequenziell organisierte $\mathrm{Ge}$ genstand sequenzanalytischer Verfahren. Mit Einschränkung gilt dies auch für Formen geronnener Handlungen, wie zum Beispiel Wohnungsarrangements. ${ }^{8}$

Ohne an dieser Stelle die Interpretationsmethode und ihre theoretische Fundierung im einzelnen zu erörtern, wollen wir die für familiengeschichtliche Gespräche gewählte Vorgehensweise im folgenden in gebotener Kürze darstellen.

(1) Zunächst werden die Sozialdaten der Familie als weitgehend ,objektive' Daten zusammengestellt und in sequenzieller Abfolge interpretiert. ${ }^{9}$ Dabei entwerfen wir für die mit den Daten bezeichneten Stationen der Entwicklungsgeschichte Entscheidungsspielräume der Familie und vergleichen diese mit den tatsächlich getroffenen Entscheidungen. Wir erhalten so ein Entscheidungsmuster, welches auf der Folie der Entscheidungsmöglichkeiten die Besonderheit des Falles als spezifischen Selektionsprozeß bereits erkennen läßt. Dies bildet die Fallstrukturhypothese der untersuchten Familie, welche im Rahmen der weiteren Analysen systematischen Falsifikationstests unterzogen wird.

(2) Es wird die Eröffnungssequenz des Textes sequenzanalytisch interpretiert im Hinblick auf die Selbstpräsentation der Teilnehmer sowie auf die pragmatische Einbettung des Gesprächs in den Familienalltag, wie sie von den Teilnehmern vorgenommen wird. ${ }^{10}$

(3) Für eine Synopse der im Familiengespräch thematisierten Bedeutungsfelder wird deren sinnlogische Abfolge - dabei insbesondere die Übergänge und Schaltstellen -, sowohl nach thematischen als auch nach interaktionsstrukturellen Merkmalen untersucht. "

${ }^{8}$ Bei der Anwendung auf andere Typen von Protokollen sozialer Phänomene ist zu beachten, daß diese in das Modell von interaktionell konstituierten Texten eingerückt werden.

${ }^{9}$ Als ,objektive' Daten betrachten wir ganz pragmatisch jene, deren Bedeutung von der Ausdrucksform im Gespräch ablösbar und damit nur gering interpretationsbedürftig ist. (Dazu gehören Zeit- und Ortsangaben zur Bildungs- und Lebensgeschichte, Berufsangaben, Bildungsstationen und weitere Sozialdaten wie Geburt, Heirat, Tod, etc.)

${ }^{10}$ Wie eine Familie die Aufgabe einer sozialwissenschaftlich induzierten familiengeschichtlichen Erzählung löst, sagt schon viel über ihren Individuierungsgrad als Fall und damit über ihre Fallspezifik aus. (Vgl. zur Bedeutung solcher Eröffnungssequenzen im Hinblick auf die Fallpräsentation aus ethnographischer Sicht C. O. Frake 1980.)
(4) Nun schließen sich weitere Sequenzanalysen nach Maßgabe immanent, das heißt: angeleitet durch den inneren Kontext des Falles, formulierter Kriterien an. Einzelne Erzählungen und Interaktionszüge werden im Hinblick auf die Reproduktionsgesetzlichkeit von Fallstrukturaspekten analysiert. Ziel dieser Detailanalysen ist es, Schritt für Schritt die Entfaltung der Fallstruktur in den unterschiedlichsten thematischen Zusammenhängen herauszuarbeiten, um die familienspezifischen, einzelerlebnisübergreifenden Deutungen in der Bearbeitung familienbedeutsamer Ereignisse zu rekonstruieren.

(5) Abschließend werden die rekonstruierten familienspezifischen Fallstrukturen in theoriebildender Absicht miteinander kontrastiert, wobei die Kontrastierungslinien sich ergeben aus dem, was die durchgeführten Fallrekonstruktionen vorzeichnen. ${ }^{12}$

\section{Zusammenfassende Darstellung einer sequenziell durchgeführten Textanalyse ${ }^{13}$}

Der Analyse liegt ein Interaktionsausschnitt aus einem Familiengespräch mit einer Bauernfamilie zugrunde. ${ }^{14}$ Gesprächsteilnehmer waren Robert Krüger, der 30jährige Jungbauer und Hofbesitzer, Dora Krüger, seine Mutter, seine Tante Irma (die ältere Schwester seines Vaters), sodann seine Schwester Claudia mit ihrem Mann Albert, seine Frau Martina und deren Eltern Herr und Frau

${ }^{11}$ Dieser Schritt könnte zu einer strukturell-inhaltlichen Beschreibung im Sinne von Schütze (1976) expandiert werden. Diese Vorgehensweise verbindet aber einen thematischen Überblick über jeweils vorliegende biographische Narrationen mit einer Strukturanalyse des Erzählaufbaus. Diese enge Kopplung kann jedoch das Ziel der Rekonstruktion einer Fallstruktur, die den Gesprächsverlauf erst motiviert, so nicht erreichen, sondern beschränkt sich in der Regel auf Analysen auf der Ebene der Gesprächsstruktur.

12 Vgl. dazu die Ausführungen zur ,strukturalen' Generalisierung in Kapitel 5.

13 Jede Darstellung einer Sequenzanalyse ist mit dem Problem belastet, einerseits anhand eines kurzen Interaktionsausschnittes das Ganze einer Fallstrukturhypothese schlüssig zu entwickeln, andererseits aus darstellungstechnischen Gründen nicht den gesamten ProzeB der Datenanalyse mit seinen Wegen und Umwegen abbilden zu können, sondern eine Auswahl zu treffen, die sich auf die markanten der verworfenen Deutungen und auf die wesentlichen Elemente der letztlich herausgearbeiteten Fallstrukturhypothese bezieht.

${ }^{14}$ Eine Einzelfallstudie der Familie „Krüger" ist im Arbeitsbericht zum erwähnten Forschungsprojekt (Hildenbrand und Oevermann 1987) dokumentiert. 
Bender. (Heinz Krüger, der Vater von Robert, lag zur Zeit des Familiengesprächs im Krankenhaus.) Auf dem Krüger-Hof leben drei Generationen: die Familie Robert Krüger mit den Kindern Stefan und Bernhard, 7 und 4 Jahre alt, sowie die Eltern von Robert, Dora und Heinz und die Tante Irma.

In der zusammenfassenden Darstellung des Interpretationsprozesses wird das Augenmerk auf die Analyse der Erzählfiguren im Hinblick auf das Thema „Gemeinsames Erzählen" gerichtet, wobei die Teilhabe der Gesprächsteilnehmer an binnenfamilialen Bedeutungsfeldern sowie an deren interaktiv hergestellten Darstellungsformen im Mittelpunkt der Rekonstruktion stehen.

\section{Die Selbständigkeit der Kinder: eine Rechtfertigung}

Martina $^{15}$ ja die Kinder meines Erachtens sind auch schon sehr selbständig also unser der $\mathrm{O}$ beide eigentlich die waren zwei Jahre alt die hab ich hier aufm Hof O laufen lassen da braucht ich keine Angst haben dasse mir auf die Straße gehen (,)

Interv1 mhm

Martina da is kein Hoftor zu und gar"nichts (,) das $O$ irgendwie als ob die das spüren" oder so $\mathrm{O}$ ma kann" nit immer hin da hinterher sein (,)

Interv1 $\mathrm{mhm}$

Mit der ,Selbständigkeit der Kinder', dem propositionalen Gehalt der Äußerung, thematisiert Martina gegenüber einem Dritten, nicht dem Familienmilieu angehörenden Adressaten eine Situation unter dem Gesichtspunkt einer lebenszyklischen Konstellation. Die Selbständigkeit der Kinder bedeutet für eine Familie immer einen entscheidenden Schritt innerhalb der Erziehung, wobei die normative Einstellung zu diesem Topos fallspezifisch variieren kann, je nachdem, welche Bedeutung diesem Erziehungsziel innerhalb der familialen Sozialisation zugemessen wird.

In der Formulierung ,die Kinder" kommt die verwandtschaftliche Beziehung zu diesen nicht zum Ausdruck.

${ }^{15}$ Liste der verwendeten Transkriptionszeichen:

Unterbrechungen: $\mathbf{O}=$ kurzes Absetzen;

Intonation: $()=$, fallend; $(-)=$ in der Schwebe; (') = steigend; gar"nichts $=$ Betonung der dem " voraufgehenden Silbe (o. Wort)
A immer hin da
B $m h m=$ gleichzeitiges Sprechen (kursiv)
$(\ldots)=$ unverständlicher Äußerungsteil

$(($ lachend: $))=$ Beobachterkommentar
Dies könnte eine Kindergärtnerin in einem Gespräch über die Kinder ihrer Gruppe gesagt haben, genausogut aber auch eine Mutter, wenn der Beziehungsmodus durch den Kontext abgedeckt ist. Ein fiktives Beispiel: Martina kommt abends aus der Singstunde nach Hause und fragt: ,Wo sind die Kinder?` ,Robert antwortet: ,Im Bett.'

Die einzelnen Darstellungspartikel in der Äußerung geben uns Aufschluß über den konkret vorliegenden Kontext des Themas. Mit ,sehr selbständig" wird die Selbständigkeit gegenüber der Normalität hervorgehoben, während mit "auch schon“" das frühe Erreichen des Entwicklungszieles vergleichend betont wird. Die Eröffnung des Satzes mit ,ja" bildet die Verknüpfung mit dem voraufgehenden thematischen Rahmen und verweist damit auf die argumentative Einstellung des Sprechers, für dessen Äußerung in diesem Rahmen mindestens zwei Sinnhorizonte vorstellbar sind: In dem einen Fall wäre eine Fraglichkeit von bestimmten Erziehungsmaximen hintergrundsthematisch, in dem anderen Fall stände das Argument in einem funktionalen Zusammenhang mit einem Handlungskreis, der mit einem bestimmten Erziehungsmodell nicht in Einklang zu bringen ist.

Bevor diese so allgemein formulierte Vermutung expliziert wird, wenden wir uns der Gestalt der sprachlichen Realisierung des Sinnzusammenhangs zu. Mit „unser“ beginnt Martina zunächst mit einem Kind, das als einer Gemeinsamkeit zugehörig bezeichnet wird: der Gemeinsamkeit der Eltern. Es wird so mit der Relation der Eltern zu einem Kind begonnen. Durch die Korrektur durch „beide eigentlich" im Sinne einer Vermeidung der Herausstellung eines von beiden wird die Möglichkeit, sich auf ein einzelnes, konkretes Ereignis zu beziehen, verschlossen. Diese Selektion präformiert die Form der Erzählung. Wenn Martina nun von beiden Kindern sprechen will, hat sie immer schon eine Verallgemeinerung eines konkreten Ereignisses vorgenommen, wenn man unterstellt, daß nicht über ein Ereignis gesprochen wird, an dem beide Kinder gleichermaßen und gleichzeitig beteiligt gewesen sind. Mit dieser Einleitung hat Martina die Darstellungsform für das noch zu thematisierende Ereignis in einer Weise präformiert, die im Hinblick auf die Veranschaulichung der Selbständigkeit auf eine konkrete Geschichte verzichtet. Gleichwohl beginnt sie mit einer Illustration ihres voraufgegangenen Arguments. Liest man „unser" im Hinblick darauf als Hervorhebung der unmittelbaren Familienzugehörigkeit, so konnte sie mit "die Kinder" durchaus auf eine milieuspezifisch gekennzeichnete Kohorte (etwa: 
alle Bauernkinder) Bezug genommen haben, die sich im Vergleich zu Kindern aus anderen Milieus im Hinblick auf die Selbständigkeit unterscheiden.

Den ersten Hinweis auf die frühe Selbständigkeit der Krüger-Kinder erhalten wir durch die Altersangabe „Zwei Jahre“ und die Schilderung „aufm Hof laufen lassen". Es wird ein Sachverhalt thematisiert, der auf beide Kinder zu unterschiedlichen Zeitpunkten nur zutreffen kann und damit auf ein allgemeines Phänomen verweist. Selbständigkeit bedeutet in diesem Zusammenhang unbeaufsichtigtes "laufen lassen" der Kinder angesichts der Gefahr, die mit dem "auf die Straße gehen" verbunden ist. Mit "da braucht ich keine Angst“ thematisiert Martina ihre Stellung zu der möglichen Gefahr. Sie antizipiert diese, kann allerdings entweder ihren Kindern vertrauen, da $\beta$ sie sich dieser Gefahr nicht aussetzen, oder es sind äußere Vorsichtsmaßnahmen getroffen worden, die diese Gefahr bannen: entweder durch Beaufsichtigung oder durch äußere Formen der Sicherung. „Lassen" spricht gegen eine Beaufsichtigung, bleibt also als naheliegende Vermutung nur eine äußere Sicherungsmaßnahme. Genau diese wird aber mit "da ist kein Hoftor zu und gar nichts" in Abrede gestellt. Erst mit diesem Äußerungsteil erhält die Behauptung der Selbständigkeit ihre fallspezifische Gestalt und Motivierung.

Die Motive des Verhaltens der Kinder bleiben Martina scheinbar rätselhaft, wie die Worte ,irgendwie äh als $o b$ die das spüren oder so" zeigen. Sie beschreibt hier in einer vagen Formulierung ein Verhalten mit dem Hinweis auf einen Empfindungshorizont, gleichsam auf einen ,sechsten Sinn der Kinder. Entscheidend ist hier der Referent für „das“. Was könnten die Kinder „spüren“? Ein möglicher Referent auch im Zusammenhang mit der Semantik von "spüren“ wäre die fehlende Angst, bzw. das Vertrauen in die Kinder. Zu spüren, daß sie nicht auf die Straße gehen dürfen, sollen, etc. setzte voraus, daß die Kinder unausgesprochene Verbote ,spüren'.

„Man kann nit immer hin da hinterher sein" scheint im bisher rekonstruierten Zusammenhang völlig aus dem Rahmen zu fallen. Was hat dieser Sachverhalt mit der Selbständigkeit der Kinder zu tun? Sollte er der Referent für das voraufgehende „das" sein, so käme zum Ausdruck, daß die Kinder spüren, daß man nicht immer hinter ihnen her sein könne, was ihre Selbständigkeit motivierte.

Doch betrachten wir zunächst den letzten Teil der Äußerung im Zusammenhang genauer. ,Nicht-hin- terher-sein-können' bedeutet zum einen, durch andere Aktivitäten daran gehindert zu sein, obwohl man es gern tun würde. Zum anderen wird dadurch gerade die Selbständigkeit eines Kindes in Abrede gestellt. Wenn die Kinder selbständig wären, brauchte man nicht ,hinter ihnen her' zu sein. Martina verwendet aber in diesem Zusammenhang das Verb ,können', und verleiht der Äußerung die objektive Bedeutung einer Rechtfertigung.

Diese Interpretation des letzten Satzes gibt einen klaren Hinweis auf den Sinnzusammenhang, der eine Rechtfertigung impliziert: Mit dem unpersönlichen „man“ distanziert sich Martina von dem Inhalt mit Verweis auf eine sozial geltende Norm. Mit ,Nichtkönnen' wird ein implizierter Verweis auf die Maxime, den Kindern gegenüber zu Betreuung und Fürsorge sich verpflichtet zu fühlen, ausgedrückt. Denn nur unter dieser Voraussetzung ergibt das wie eine Entschuldigung formulierte „ma kann nit immer hin da hinterher sein" einen Sinn. Wenn jedoch in der Form einer Rechtfertigung eine Erziehungsmaxime thematisiert wird, so kann dies entweder als Reaktion auf selbstgesetzte Ansprüche geschehen, die durch reale Umstände zurückgedrängt werden. Oder aber dies erfolgt mit Bezug auf einen binnenfamilialen Bedeutungsraum, in dem dieses Thema Konfliktpotentiale birgt, gegen welche durch eine vorgreifende Stellungnahme dazu in Form einer Rechtfertigung die eigene Position gesichert werden soll. Schon bis zu diesem Punkt läßt sich unschwer erkennen, daß das Thema der Selbständigkeit der Kinder verknüpft wird mit einem Anspruch, welcher mit der Betreuungsverpflichtung den Kindern gegenüber in Konflikt steht. Vergegenwärtigt man sich potentielle Hinderungsgründe für die nicht realisierbare Überwachung der Kinder, so kommt man auf die insbesondere für bäuerliche Milieus charakteristische spezifische Ausgestaltung der Arbeitsrolle und damit auf das Verhältnis von Arbeits- und Erziehungsaufgaben in bäuerlichen Familien. Die Rechtfertigung ergibt sich nun daraus, da $\beta$ für Martina die Selbstverständlichkeit traditionaler, für das bäuerliche Milieu angemessene Erziehungsstile nicht gilt. Selbst aufgewachsen in einem städtischen Angestelltenhaushalt, versucht sie, den Ansprüchen eines urbanen, auf der Trennung von Arbeits- und Erziehungsbereich aufbauenden Erziehungsmodells zu folgen, die im bäuerlichen Familienbetrieb so nicht vorliegt.

An Martinas Äußerung schließt Robert an:

Robert sind natürlich auch immer im Bauen aufgewachsen 
Die Tatsache, daß Robert an dieser Stelle ${ }^{16}$ mit einer impliziten Erklärung für das Verhalten der Kinder aufwartet, läßt vermuten, daß es sich hier um ein die Kernfamilie betreffendes Thema handelt, welches Robert insbesondere für das Verständnis der Milieufremden einer Erläuterung für bedürftig hält. Indem er das Milieu der Kinder mit „im Bauen“ umreißt, thematisiert er ein Provisorium, welches für Kinder Gefahren birgt, sie aber auch in der Meisterung dieser Gefahren Erfahrungen machen läßt, die durchaus zu Selbständigkeit beitragen können. Mit „natürlich“ wird dieser Sachverhalt als Selbstverständlichkeit bekräftigt, die die vage „als ob"-Formulierung von Martina konkretisiert. Robert nimmt damit Martinas thematischen Fokus auf und ergänzt ihre Darstellung. Mit ,immer" verweist Robert auf eine Permanenz des Provisoriums (,im Bauen“), das dadurch gleichsam zur Normalsituation erklärt wird. Weder „spüren“ die Kinder das Gefühl der Mutter (keine Angst), noch realisieren sie ihr ,Nicht-hinterhersein-können', sondern sie haben in der von Robert näher qualifizierten Lebenssituation (,,im Bauen“) gelernt, sich selbständig zu verhalten. Die Thematisierung des Provisoriums nimmt gleichzeitig in einer entlastenden Form Martinas Rechtfertigungsintention auf. Die Eltern, mit Bauen beschäftigt, haben dadurch weniger Zeit für die Aufsicht der Kinder. Für Robert steht allerdings die Situation der Kinder im Vordergrund, weniger der besondere Konflikt, den Martina mit ihrer Rechtfertigung zum Ausdruck bringt.

Vergleicht man Martinas Qualifikation der sozialisatorischen Einstellung zu den Kindern mit Roberts Ergänzung, dann wird die Differenz deutlich: Robert kann sich in die Erfahrungswelt der Kinder problemlos hineinversetzen und thematisiert mit „im Bauen" den Binnenbereich des Hauses und des Hofes, in dem die Kinder trotz der Gefahren gelernt haben, sich wie selbstverständlich zu bewegen, während Martina mit der Thematisierung des Hoftors und der Straße das Überschreiten der Grenzen von Haus und Hof und damit der Grenzen ihres Aufsichtsbereiches zum Bezugspunkt ihrer Darstellung macht.

16 Vor der Interpretation der sich jetzt anschließenden Äußerung wären zunächst die pragmatisch sinnvollen Anschlußmöglichkeiten aller Anwesenden zu entwerfen, um die Äußerung selbst als eine fallspezifische Selektion charakterisieren zu können. Die Ergebnisse dieses Schrittes werden im folgenden integriert.
Es wird im folgenden gezeigt werden, wie Robert seine Perspektive nicht nur in der thematischen Auswahl, sondern auch in der Darstellungsform seiner Geschichten zum Ausdruck bringt.

Martina nimmt Roberts Rede vom Provisorium auf und ergänzt sie mit der fragmentarischen Schilderung von wechselseitig konkurrierenden Handlungsabläufen.

Martina ja immer aufhören (...) und dann erstmal woanders hin dazwischen aber

Robert irgendwo war immer $n$ Loch $\mathrm{O}$ wo sie aufpassen mußten

((mehrstimmiges Lachen))

Martina gibt im Kontext ihrer Rechtfertigung nun ihrerseits eine Erläuterung dafür, in welcher Weise die überschneidenden Handlungskreise sich auswirken. Sie wird allerdings von Robert mit einer seinen ersten Einwurf ergänzenden Situationsbeschreibung, gleichsam als Kommentar zum bisher Gesagten, unterbrochen. Robert bezieht sich im Gegensatz zu Martina, die ihren Konflikt zum Ausdruck bringt, explizit auf die Kinder. Der verallgemeinernde Charakter des Satzes, von dem wir vermuten, daß er jenem Typ von Sätzen zurechenbar ist, welcher für Ginzburg die Grundlage familialer Gemeinsamkeit bildet, öffnet eine Vielzahl von Anschlußmöglichkeiten, deren Implikate bei den Anwesenden Heiterkeit auslösen. In jedem Fall bildet der Satz als generalisierende Feststellung den Versuch einer Zäsur im Erzählgeschehen. Bereits an dieser Stelle zeichnet sich mit der Äußerung des ,Loch-Satzes' die fallspezifische Interaktionsstruktur der Familie Krüger ab, deren Reproduktion nun weiter verfolgt werden soll.

\section{Roberts Anschlußerzählung}

Robert jo das $O$ der zum Beispiel hat der Kleine hier $O$ is vier geworden ja $O$ und ham we voriges Jahr im Winter hier die Treppe gemacht $O$ du warst ja auch da $O$ hat der im Bett gelegen und da hab ich also hier hat er geschlafen

Martina das können Sie sich garnicht vorstellen

Robert und hier war die Wand und da hab ich die Wand abgeklopft da hat er geschlafen

Martina hat er nich gemerkt das (-)

Robert hat er (-)

Martina wirklich also gar"nichts ((lachend:)) ja

Robert wählt ein prägnantes Beispiel für seine allgemeine Erklärung (,im Bauen aufgewachsen") und detailliert damit die schon durch ,natürlich“ angedeutete Einschätzung der Situation als veralltäglichter. Das Beispielhafte der Episode wird als 
Rahmung markiert, „der Kleine“ wird durch die Altersangabe charakterisiert. Im Gegensatz zu Martina wählt Robert die Schilderung eines konkreten Ereignisses in seiner Bedeutung für die Kinder (nicht wie Martina, die einen eigenen Konflikt zum Ausdruck brachte). Er unterbricht seine Erzählung durch den Einschub „du warst ja auch da". Damit wird der Adressat dieses Einschubes, sei dies in der Funktion von Zeugenschaft oder als Aufforderung zur Ergänzung der Darstellung, in das Kollektivsubjekt der Erzählung eingereiht. In der Erzählung spricht er von „wir“, als er den Treppenbau erwähnt. Würde damit die Anwesenheit in der konkreten Situation thematisiert, so hieße es: „du warst ja auch dabei“. So aber muß sich die Äußerung auf einen Sachverhalt beziehen, dessen konstitutive Bedingung die nur gelegentliche Anwesenheit bildet. Dieser kann auf zwei Bedeutungsebenen zum Ausdruck kommen. Das Nächstliegende wäre der Bezug auf alle nicht im Haus lebenden und an dem Familiengespräch teilnehmenden Personen außer den Interviewern. Bezöge sich die Äußerung auf ständig im Haus lebende und damit dem Haushalt zugehörige Personen, die der strukturellen Bedingung einer ständigen Anwesenheit genügten, wie dies insbesondere für Martina im Hinblick auf ihre Aufgaben in der Kindererziehung als Mutter gilt, so wäre damit ein Strukturproblem in dieser Familie implizit angesprochen.

Für beide Bedeutungsebenen gilt allerdings in erzähltheoretischer Hinsicht, daß die Thematisierung der Anwesenheit ihre Relevanz auf der einen Seite als Markierung der Erzählung als eine in der Familie schon bekannte Geschichte gewinnt. Auf der anderen Seite werden die beim Gespräch Anwesenden hinsichtlich des Erzählraums klassifiziert in solche, die dem Erzählraum und damit dem Zentrum des Familienmilieus als zugehörig zu betrachten sind, und in solche, die als Fremde Adressaten der Erzählung selbst sind. Diese Zuordnung zeigt, daß, typisch für einen traditionalen dörflichen Lebenszusammenhang, eine Kategorie für ,neutrale Dritte', die weder als kollektives Erzählsubjekt dem Erzählraum angehören noch als Adressat der Erzählung markiert sind, nicht verfügbar ist. ${ }^{17}$

${ }^{17}$ Dies zeigt schon die einfache Beobachtung, daß man, wenn man als Fremder durch ein Dorf geht, in der Regel gegrüßt wird. In der Stadt hingegen, in welcher die Kategorie des Fremden als ,neutralem Dritten' zur Selbstverständlichkeit gehört, ist dies nicht der Fall.
Mit ihrem begleitenden Kommentar („das können Sie sich garnicht vorstellen“), der in gesprächsorganisatorischer Hinsicht als Dramatisierung von Roberts Erzählung gelesen werden kann, verleiht Martina der Erzählung die Qualität des Außerordentlichen. Damit läßt sie erkennen, daß sie weiß, was Robert erzählen will, und daB sie die Pointe der Geschichte kennt. ${ }^{18}$ Robert hat also mit seiner Erzählung und durch die Abgrenzung von Vertrautheit und Fremdheit den strukturell bestehenden Raum von familialer Gemeinsamkeit evoziert, den Martina mit ihrem Einschub bekräftigt. Was zunächst wie eine parallele Bekräftigung Martinas, Roberts Erzählung betreffend, aussieht, wird dann zu einer Vorwegnahme der Pointe von Roberts Geschichte, der damit um den Erfolg gebracht ist.

Die eine Lesart wäre, daß Martina damit eine Konkurrenz mit Robert zum Ausdruck bringt. Martinas Beteiligung ließe sich mit der als implizite Aufforderung wirkenden Grenzziehung von Robert motivieren. Die Aufforderung bedeutet aber nicht zwangsläufig die Vorwegnahme der Pointe. Die Reaktion Martinas verwiese so auf einen latenten Vorwurf, der mit dem Einschub von ,du warst ja auch da" erhoben worden wäre. Indem sie die Beendigung der Geschichte übernimmt, zeigt sie ihre Teilhabe am gemeinsamen familiären $\mathrm{Er}$ fahrungskreis, weist aber einen latenten Vorwurf zurück, dessen Möglichkeit sie damit prinzipiell anerkennt.

Die andere Lesart wäre: Es handelt sich hier um ein anschauliches Beispiel für eine Form gemeinsamen Erzählens. Eingeleitet durch Roberts Zuordnung der Beteiligten, gleichsam als Regieanweisung an die Darstellungsform der Erzählung, entsteht unter Beteiligung von Martina eine gemeinsame Erzählung. Die Frage stellt sich jedoch, inwiefern hier dennoch ein konkurrierendes Element wirksam wird, wenn man unterstellt, daß Martina Robert die Pointe vorwegnimmt und ihm damit seinen Sinn der Geschichte unter der Hand zu ihrem eigenen umwandelt.

${ }^{18}$ Die Tatsache, daß Martina sich zu Wort meldet im AnschluB an Roberts Einschub „du warst ja auch da“, läßt die Vermutung $z u$, daB sie die latente Bedeutungsebene (das Strukturproblem in der Ehebeziehung, welches sich auf die Aufgabenverteilung in der Kindererziehung bezieht) wahrnimmt. 


\section{Roberts thematische Detaillierung:} Selbständigkeit und Fürsorge

Robert da ging der auch nit alleine die Treppe runter weil er genau wußte das is $\mathbf{n}$ Loch da $\mathrm{O}$ fall ich nachher noch runter und da hab ich ihn immer die Treppe runter getragen

Interv1 $\mathrm{mhm}$

Handelte die erste Episode von der Unbeeindruckbarkeit des Sohnes durch die laute Arbeit an der Wand, so steht jetzt das gegenseitige Vertrauen zwischen Vater und Sohn im Vordergrund. Es basiert auf der Selbständigkeit des Sohnes im Wissen um die Gefahren, während seine objektive Hilfsbedürftigkeit vom Vater zuverlässig aufgenommen wird, wobei jetzt die Verläßlichkeit des Vaters in den Mittelpunkt rückt. Mit ,und da hab ich ihn immer die Treppe runter getragen“ betont Robert seine fürsorgliche Haltung gegenüber seinem Jüngsten in ereignisübergreifender Form. War der Fokus in der ersten Episode seiner Erzählung noch auf eine Eigenschaft seines Kindes gerichtet, so verschiebt sich nun der Fokus auf Roberts Aktivität und hebt so die Betreuungsthematik in der Erweiterung des Themas ,Selbständigkeit' auf die explizite Ebene der Darstellung. Vergleichen wir diese Coda mit der von Martina, so fällt auf, daß sie mit ihrem Schlußsatz ,ma kann nit immer hin da hinterher sein“ ihren Konflikt zwischen Arbeits- und Erziehungsverpflichtung betont, während Robert die für ihn unproblematische Fürsorge ausdrückt. Robert schildert damit eine zu Martina im Gegensatz stehende Konsequenz aus der durch das Bauen gekennzeichneten Situation: Unproblematisch erbrachte Fürsorge und nicht durch die Umstände erzwungenes ,Laissez-faire' bestimmt die sozialisatorische Handlungspraxis.

Robert

das gab da $\mathrm{O}$ er stand dann auf und hat er gerufen $O$ Mama oder Papa hol mich und da wars gut $O$ da brauchten se keine Angst zu haben $\mathrm{da} B$ der irgendwo reinspringt

Robert korrigiert im Anschluß seine Schilderung in zwei Punkten. Der Sohn ruft die Eltern zu Hilfe - man könnte sagen: seine Selbständigkeit besteht darin, die objektive Hilfsbedürftigkeit zum Ausdruck zu bringen. Des weiteren wird die Darstellung der Ausschließlichkeit seiner aktiven Fürsorge auf die Darstellung der Aktivität beider Eltern als gemeinsamer sozialisatorischer Instanz erweitert: „Da brauchten se keine Angst zu haben daß der irgendwo reinspringt". Dabei wird mit dem ,se“ auf eine Rechtfertigung abgehoben, die auf einer allgemeinen Ebene angesiedelt ist.
Martina hat ihre Schlußfolgerung (,da braucht ich keine Angst zu haben, daß se mir auf die Straße gehen") in einer Weise formuliert, die ihre individuelle Beziehung zur ,Gesamtheit' der Kinder zum Ausdruck bringt. Der gemeinsame Nenner ist deutlich: Man braucht um die Kinder keine Angst zu haben. Übereinstimmend auch die Deutung, $\mathrm{da}$ die Kinder um die Gefahren wissen. Der Unterschied liegt in der sprachlichen Realisierung der Konsequenz, die auf die unterschiedlichen Motive für die jeweiligen Anteile an der gemeinsamen Erzählung verweist:

Martina befindet sich in einem Konflikt, weil sie einerseits für die Kinder da sein, andererseits ihrer Arbeitsverpflichtung auf dem Bauernhof nachkommen will. Der selbstgesetzte Anspruch einer umfassenden Betreuung der Kinder gerät in Konflikt mit den Arbeitsanforderungen auf dem Bauernhof und führt zu ihrer Rechtfertigung. Einerseits orientiert sie sich an einem Erziehungsmodell, welches auf der Trennung von Arbeits- und Erziehungsaufgaben basiert, wie sie es auch aus ihrer kleinfamilialen Herkunft kennt. Andererseits hat sie sich in einer ,Nachsozialisation' die Lebens- und Arbeitsbedingungen in einer Großfamilie auf einem Bauernhof angeeignet, die eine andere Haltung angesichts der Einheit von Arbeits- und Erziehungssituation im ,ganzen Haus' erforderlich machen.

Roberts Deutung der Überflüssigkeit der Angst um die Kinder beruht auf der seinerseits als zureichend empfundenen Fürsorge und dem dadurch gesicherten Vertrauen in die Kinder. Die referentielle Vagheit in Martinas Äußerung, warum sie keine Angst $\mathrm{zu}$ haben brauche, wird in Roberts Äußerung konkret. Die oben entfaltete Differenz in der sprachlichen Realisierung verweist auf das für Robert noch wie selbstverständlich geltende ,traditionale' Erziehungsmodell, während für Martina ein Handlungsproblem aus der Konkurrenz unvereinbarer Erziehungsvorstellungen entsteht.

\section{Die Reproduktion der Interaktionsstruktur in Martinas Reformulierung des Erzählthemas}

Martina ja die waren das auch gewohnt genau wie morgens" die ham $O$ wie meine Schwiegermutter noch mit rausging

Robert ja (...)wie ich gesagt hab da is immer irgendwon Loch auf

Martina da äh konnten ja die Kinder um sechs noch nit aufstehen oder so

Interv2 $\mathrm{mhm}$

Martina die ham dann geschlafen bis um neun der Große hat teilweise $O$ neun viertel nach neun hat der gelegen hat gewartet bis ich hochkomm und hol ihn $O$ da gabs nichts

Dieser Erzählabschnitt weist mehrere Strukturhomologien zu Roberts zweiter Erzählung auf. Es ist ebenfalls die Rede von einer Interaktion zwischen 
Sprecher und Kind. Die Pragmatik der Situation handelt gleichfalls von einer Angewiesenheit des Kindes auf einen Erwachsenen: Martina schildert eine Situation, in der sie den „Großen“ holt. Die entscheidende Differenz zu Roberts Geschichte von dem „Kleinen“ besteht in zwei Punkten: a) der „Große" wartet, bis die Mutter ihn holt - der Kleine hatte sich gemeldet; b) Martinas Schlußfolgerung lautet „da gabs nichts“, während Robert mit „da wars gut" seine Situationsschilderung ganz anders bewertet. Die Perspektivendifferenz zwischen Robert und Martina reproduziert sich hier in der nämlichen Weise: Martinas „da gabs nichts“ entspricht sinnlogisch der Einstellung „ma kann nit immer hin da hinterher sein".

Martina nimmt das latente Thema ihrer ersten Geschichte in neuer Form auf, indem sie eine weitere Person einführt: Als die Schwiegermutter sich an der Feldarbeit beteiligte („mit rausging“), stellte sich das Problem der Vereinbarung von Kinderbetreuung und Feldarbeit verschärft. Martina wird an dieser Stelle von Robert unterbrochen, der mit der Wiederholung seines ,Loch-Satzes' darauf verweist, daß es sich jetzt um eine überflüssige Wiederholung des vorher abgehandelten Themas handelt. Er hält seinerseits das Thema für erschöpfend besprochen und nimmt zum Inhalt nicht mehr Stellung, sondern reagiert ungeduldig mit der inzwischen sich als ,Standardsatz' etablierten Bemerkung.

Daß Martina ihre Geschichte als ,Parallelgeschichte' und in Konkurrenz zu Robert erzählt, zeigt die Rahmung: ,genau wie morgens". Ihre Absicht, mit Robert, gleichzuziehen' und einen Beleg für ihre Erzählperspektive zu liefern, gerät zu einer Reproduktion der Darstellung von einer Unselbständigkeit der Kinder, die in sichtlichem Gegensatz zu der einleitenden Thematik, die Selbständigkeit der Kinder, steht. An dieser Stelle setzt Dora ein:

$\begin{array}{ll}\text { Dora } & \text { jo O und wo wir dann fertig waren } d a \beta \text { wir } \\ & \text { Kaffee } \\ \text { Robert } & \text { ja }=\text { ja } \\ \text { Dora } & \text { getrunken hatten }(. . .)(. .) \\ \text { Martina } & \text { ja und dann"wurde das Kind geholt } \\ \text { Robert } & \text { haben wir die Kinder geholt }\end{array}$

Die Schwiegermutter Dora assistiert Martina, indem sie eine Ergänzung zu Martinas Erzählung anführt. Durchaus affirmativ mit ,jo“, erläutert sie, warum das Kind bis neun Uhr warten mußte. Das Kaffeetrinken der Erwachsenen nach dem ersten frühmorgendlichen Arbeitsgang hat Priorität vor der Versorgung der Kinder. Dora repräsentiert hier die traditionalen Erziehungsvorstellun- gen. Robert kommentiert die Äußerung seiner Mutter mit einem kurzen , $j \mathrm{ja}=\mathrm{ja}$ “, noch bevor sie zu Ende gesprochen hat. Martina hakt ebenfalls sofort ein. Beide sind sich zumindest darin einig, $\mathrm{da} \beta$ die Schwiegermutter $z \mathrm{u}$ dem verhandelten Thema nichts wesentlich Neues beitragen könne. Dieser gesprächspragmatisch vollzogene Ausschlu $\beta$ bedeutet, da $\beta$ es beiden gelungen ist, in dieser bäuerlichen Haushaltsfamilie ein eheliches Subsystem auszubilden. Während Robert mit ,ja$=$ ja" kenntlich macht, daß Doras Beitrag am verhandelten Thema der Aufgabenverteilung zwischen den Eltern in der Kindererziehung vorbeizielt, knüpft Martina mit ,ja und dann" wieder an das Thema an und wiederholt den Schluß der eigenen Geschichte, indem sie eine Bedeutungstransformation in der Sachverhaltsdarstellung vornimmt und damit einem möglichen Einwand begegnet. In der Bewegung von ,ich hochkomm und hol ihn" zu ,dann wurde das Kind geholt" wird der Aktor der Handlung getilgt. Der Einwand, dem Martina damit aus dem Weg geht, bezieht sich auf den Sachverhalt, wer nun tatsächlich das Kind geholt hat. Weder die Schwiegermutter noch Robert können nun begründet ihr widersprechen und behaupten, daß sie es seien, die ,das Kind holen'. Gleichzeitig mit Martina knüpft auch Robert an die Äußerung seiner Mutter an und bringt seine Deutung des Sachverhalts: ,haben wir die Kinder geholt". Der Vergleich mit Martinas Satz zeigt uns folgendes: Während Martina den Aktor tilgt, erweitert Robert zu „wir“; hatte Martina nur von einem Kind gesprochen, so thematisiert Robert beide Kinder. Robert vollzieht hier die gleiche Sinnbewegung in der Deutung des Sachverhalts wie am Ende seiner eigenen Schilderung: er erweitert die Perspektive einer individuellen Beziehung (Martina zum Großen; Robert zum Kleinen) auf eine Perspektive, in der die Kinder gemeinsam Gegenstand sind.

\section{Die besondere Gestalt des Einigungsprozesses zwischen Martina und Robert}

Martina also der lag dann von abends $O$ bis zum anderen Morgen $O$ mittags kam er dann auch noch $O$ das ging" eben (.) naja der Zweite dann $O$ das is doch schon wieder was anderes dann wenn zwei Kinder da sind ne dann macht der Krach und der andere hörts (.) aber äh

Robert ((leise)) der Zweite hats ohnehin (...)

Martina die Kinder sind" das eben gewöhnt daß man nich so viel für sie hat $\mathbf{O}$ oder müssen sich dran gewöhnen $O$ kennen eben nix anders (.)

Robert die kennens nich anders (.) 
Martina reformuliert mit dem resümierenden ,also" als Rahmung ihre Geschichte und thematisiert die Differenz zwischen der Situation mit einem Kind und der mit zwei Kindern. Sie erweitert den thematischen Fokus um die Probleme, die bei zwei Kindern entstehen: der Betreuungsaufwand steigt und damit verschärft sich auch der Konflikt zwischen Arbeits- und Erziehungsaufgaben. Hinzu kommt der systematisch vollzogene Ebenenwechsel, der zuletzt an Martinas Überleitung zu einer verallgemeinernden Feststellung deutlich wird. Mit „die Kinder sind das eben gewöhnt ... oder müssen sich daran gewöhnen" spezifiziert sie einerseits ihre zu Beginn so vage Formulierung mit „spüren“, andererseits begibt sie sich mit dieser Schlußfolgerung (,eben“) auf die Ebene, die jenseits einer Konfrontation durch konkurrierende Perspektiven liegt. Gleichwohl bleibt ihr impliziter Anspruch (,mehr Zeit für die Kinder haben zu wollen'), den sie einem ,modernen' Erziehungskonzept verdankt und der ihre Rechtfertigungshaltung motivierte, als Element der Einsicht in die Gegebenheiten auf dem Bauernhof erhalten. Entsprechend verwendet sie hier wieder das unpersönliche "man" (wie in ihrer allerersten Coda) und tilgt dadurch die Form der persönlichen Stellungnahme in einer distanzierenden Redeweise.

Ihre abschließende Bemerkung, „kennen eben nix anderes" wird von Robert so modifiziert, daß der Perspektivenunterschied, der die ganze Interaktionssequenz bestimmte, in seiner Ausdrucksgestalt erhalten bleibt. Durch die Betonung von „kennen“ bestreitet er die resignative Elemente beinhaltende Unterstellung in Martinas Argumentation. Das heißt, er weist implizit darauf hin, daß es sich um eine Selbstverständlichkeit handelt, die keiner besonderen Rechtfertigung bedarf.

\section{Familie als Milieu und Familie als Ort der Selbstthematisierung}

Roberts traditionale Erziehungsvorstellungen, die für ihn noch selbstverständlich sind, verlieren angesichts der veränderten Arbeitsbedingungen auf dem Hof, die die Mitarbeit seiner Frau auf dem Feld erfordern, zunehmend ihre unproblematische Geltung. Anders formuliert: Die latente Sinnstruktur der Interaktion zwischen Robert und Martina wird geprägt durch die Diskrepanz von traditionalen und ,modernen' Erziehungsvorstellungen angesichts der aktuellen Realitätsanforderungen auf einem Bauernhof. Der gestiegene Grad an Arbeitsteilung in der Landwirtschaft kollidiert zu- nehmend mit den tradierten Sozialisationsvorstellungen, ist aber mit einem städtischen Erziehungskonzept genausowenig vereinbar. Die Konkurrenz in der Darstellung der Familienwirklichkeit läßt die Konfliktpotentiale deutlich werden, die in der widersprüchlichen Einheit von traditionalen und modernen Anforderungen an die Lebenspraxis auf dem Bauernhof bestehen. Martina thematisiert die Diskrepanz, da sie über beide Erziehungsmodelle verfügt: über die urbanen Erziehungsnormen mit einer sich verselbständigenden emotionalen $\mathrm{Zu}$ wendung durch die Trennung von Arbeits- und Erziehungsbereichen, die sie von ihrer Herkunft aus einem Angestelltenhaushalt kennt, und über die bäuerlich-ländlichen Erziehungsnormen, die in der Einheit des ,ganzen Hauses' und durch die verwandtschaftliche Solidarität fundiert sind. Für Robert gelten noch die in der Familiengeschichte tradierten Erfahrungsmodi und Handlungsmaximen, wobei er mit dem Satz ,irgendwo war immer n Loch" deutlich macht, daß die Sozialisationsbedingungen von Kindern in bäuerlichen Familienbetrieben mit natürlichen Risiken behaftet sind, welche nur begrenzt kontrolliert werden können, ohne die Routine dieses Alltags insgesamt zu gefährden. "Irgendwo war immer n Loch" ist für die Familie Krüger, ohne daß sie sich dessen bewußt ist, einer jener Sätze, von denen Ginzburg gesagt hat, $\mathrm{da} B$ sie die Grundlage der familiären Einheit darstellten. Insofern ist dieser Satz für das hier verhandelte Thema nicht in erster Linie wichtig wegen seines Inhalts, sondern wegen seiner Qualität, einen Sinnzusammenhang, in diesem Fall die Sozialisationsbedingungen der Kinder in dieser weitgehend traditional verfaßten bäuerlichen $\mathrm{Fa}$ milie, ans Licht zu bringen.

Der Fortgang der ,Loch-Geschichte' zeigt, daß mit der Aktualisierung der Metapher auf einen Sinnzusammenhang abgehoben wird, dessen Bearbeitung der traditionalen Verfaßtheit des Milieus folgend vorgenommen wird: nicht die Familie als Familie wird diskursiv thematisiert (vgl. Abschnitt 2.), sondern hier wird ein Dissens erzählerisch an Erfahrungsgehalte gebunden, die der Lebenspraxis entstammen. (Vgl. Hahn 1988 sowie die kontrastiven Analysen in Hildenbrand und Oevermann aaO.)

Gegenstand dieses Aufsatzes war die Entfaltung des Arguments, daß es sinnvoll ist, einen soziologischen Zugang zum Erzählen so zu wählen, daß von vornherein auf gemeinsame Sinnbildungsprozesse abgezielt wird. In diesem Zusammenhang ist die Verallgemeinerungsfähigkeit von Fallrekonstruktionen kurz anzusprechen. Was das Verhältnis des 
Besonderen eines Einzelfalls zum Allgemeinen betrifft, so ist festzuhalten, daß es sich hier nicht um eine empirische Generalisierung i. S. eines Schlusses von $n$ auf $n+1$ handelt. Ein Einzelfall ist unserem Verständnis zufolge ein Allgemeines insofern, als er im Kontext allgemeiner sozialer Regelhaftigkeiten sich gebildet hat. Und er ist ein Besonderes insofern, als er sich im Kontext dieser allgemeinen Bedingungen in spezifischer Weise individuiert hat. Diesem dialektischen Verhältnis von Allgemeinem und Besonderem wird bei fallrekonstruktiven Verfahren in der Weise Rechnung getragen, da $B$ fallkontrastierend vorgegangen wird.

Die Strukturale Hermeneutik geht bei Fallrekonstruktionen generell so vor, daß zu jedem Fall gedankenexperimentell jene Fälle entworfen werden, die der vorliegende Fall auch hätte werden können, aber nicht geworden ist. (Oevermann 1985) Wir ziehen es vor, systematisch im Sinne eines „theoretical sampling“" (Glaser/Strauss 1973 und Star/Strauss 1986) Material verschiedener, auf der Basis eines jeweils Gemeinsamen möglichst kontrastierender Fälle zu erheben, um auf diese Weise zur Bildung von Typen zu gelangen. ${ }^{19}$ Strukturale Hermeneutik und Grounded Theory unterscheiden sich in der wissenschaftstheoretischen Begründung zwar erheblich, aber auf der Ebene der Forschungspragmatik hat es sich gezeigt, daß beide Ansätze, solange es um die materiale Analyse geht, sinnvoll zu kombinieren sind.

\section{Literatur}

Bausinger, H., 1958: Strukturen alltäglichen Erzählens. Fabula 1: S. 239-254.

Beck, U., 1986: Die Risikogesellschaft. Frankfurt: Suhrkamp.

Berger, P. L./Kellner, H., 1965: Die Ehe und die soziale Konstruktion der Wirklichkeit. Soziale Welt 16: 220-234.

Bergmann, J., 1985: Flüchtigkeit und methodische Fixierung sozialer Wirklichkeit. S. 299-320 in: W. Bonß/ Hartmann, H. (Hg.), Entzauberte Wissenschaft, Sonderband 3 der Sozialen Welt. Göttingen: Schwartz.

Blankenburg, W./Hildenbrand, B. et al., 1983: Familiensituation und alltagsweltliche Orientierung Schizophrener. Unveröff. Abschlußbericht für die DFG. Marburg.

Boatright, M. C., 1958: The Family Saga as a Form of Folklore. S. 1-19 in: M. C. Boatright, R. B. Downs, J. T. Flanagan, Saga and other Phases of American Folklore. Urbana.

${ }^{19}$ Hinsichtlich eines Beispiels vgl. Hildenbrand (1985)
Brose, H.-G./Hildenbrand, B., 1988: Vom Ende des Individuums zur Individualität ohne Ende. Opladen: Leske und Budrich.

Frake, C. O., 1980: How to Enter a Yakan House. S. 214-232 in: Ders., Language and Cultural Description. Stanford: Stanford Univ. Press.

Ginzburg, N., 1983: Mein Familienlexikon. Frankfurt: Suhrkamp.

Glaser, B./Strauss, A., 1973: The Discovery of Grounded Theory. Chikago: Aldine.

Gurwitsch, A., 1977 (1927): Die mitmenschlichen Begegnungen in der Milieuwelt. Berlin: De Gruyter.

Hahn, A., 1988: Familie als Raum der Selbstthematisierung. S. 169-179 in: Lüscher, K. et. al. (Hg.) Die ,postmoderne' Familie. Konstanz: Universitätsverlag.

Halbwachs, M., 1966 (1925): Das Gedächtnis und seine sozialen Bedingungen. Berlin/Neuwied: Luchterhand.

Hess, R. D., Handel, G., 1975: Familienwelten. Düsseldorf: Schwann.

Hildenbrand, B., 1983: Alltag und Krankheit. Stuttgart: Klett-Cotta.

Hildenbrand, B., 1985: Familiensituation und Ablöseprozesse Schizophrener. Soziale Welt 36: S. 336-348.

Hildenbrand, B., 1987: Wer soll bemerken, daß Bernhard krank wird? - Familiale Wirklichkeitskonstruktionsprozesse bei der Erstmanifestation einer schizophrenen Psychose S. 151-162 in: J. Bergold/U. Flick (Hg.), Erforschung der Sicht des Subjekts.

Hildenbrand, B., 1988: Modernisierungsprozesse in der Landwirtschaft und ihre Bewältigung. Vergleich einer ,schizophrenen' mit einer, normalen' Familie. S. 297-311 in: K. Lüscher (Hg.), Die ,post-moderne" Familie. Konstanz: Universitätsverlag.

Hildenbrand, B./Oevermann, U., 1987: Prozesse der Wirklichkeitskonstruktion im gemeinsamen familiengeschichtlichen Erzählen. Arbeitsbericht für die DFG

Jolles, A., 1932 (1930): Einfache Formen. Tübingen: Niemeyer.

Kallmeyer, W./Schütze, F., 1976: Konversationsanalyse. Studium Linguistik 1: 1-28.

Koselleck, R., 1979: Darstellung, Ereignis, Struktur. S. 144-157 in: Ders., Vergangene Zukunft - Zur Semantik geschichtlicher Zeiten. Frankfurt: Suhrkamp.

Lehmann, A., 1978: Erzählen eigener Erlebnisse im Alltag. Zeitschrift für Volkskunde 74: 198-215.

Lehmann, A., 1980: Rechtfertigungsgeschichten - Über eine Funktion des Erzählens eigener Erlebnisse im Alltag. Fabula 21: 65-68.

Oevermann, U., Allert, T., Konau, E., Krambeck, J., 1979: Die Methodologie einer ,objektiven Hermeneutik' und ihre allgemeine forschungslogische Bedeutung in den Sozialwissenschaften. S. $352-434$ in: H.-G. Soeffner (Hg.) Interpretative Verfahren in den Sozialund Textwissenschaften, Stuttgart: Metzler.

Oevermann, U., 1981: Fallrekonstruktion und Strukturgeneralisierung als Beitrag der objektiven Hermeneutik zur soziologisch-strukturtheoretischen Analyse. Unveröffentl. Manuskript. Universität Frankfurt.

Oevermann, U., 1983: Zur Sache. Die Bedeutung von Adornos methodologischem Selbstverständnis für die 
Begründung einer materialen soziologischen Strukturanalyse. S. 234-289 in: Friedeburg, L./Habermas, J., Adorno-Konferenz 1983. Frankfurt: Suhrkamp.

Oevermann, U., 1985: Kriminalistisches Problemlösen als Strukturgeneralisierung. Elemente einer Theorie der kriminalistischen Handlungspraxis und des inneren Widerspruchs von Bürokratisierung und kriminalistischer Handlungslogik. S. 276-293 in: Oevermann, U./ Schuster, L./Simm, A., Zum Problem der Perseveranz in Delikttyp und Modus operandi. Wiesbaden: BKA.

Quasthoff, U., 1980: Gemeinsames Erzählen als Form und Mittel im sozialen Konflikt oder Ein Ehepaar erzählt eine Geschichte. S. 109-141 in: K. Ehlich, (Hg.), Erzählen im Alltag. Frankfurt: Suhrkamp.

Soeffner, H.-G., 1980: Überlegungen zur sozialwissenschaftlichen Hermeneutik am Beispiel der Interpretation eines Textausschnittes aus einem ,freien' Interview. S. 70-96 in: T. Heinze/H. W. Klusemann/H.-G. Soeffner $(\mathrm{Hg}$.$) , Interpretationen einer Bildungsge-$ schichte. Bensheim: Beltz.

Star, S. L./Strauss, A., 1986: Sampling in Qualitative Research. Ms. Tremont Research Institute.

Strauss, A., 1987: Qualitative Analysis for Social Scientists. Cambridge: Cambridge University Press. 